\title{
Prognosis-related classification and dynamic monitoring of immune status in patients with sepsis: a prospective observational study
}

Jun Yin

Zhongshan Hospital Fudan University

Yao Chen

Zhongshan Hospital Fudan University

Junling Huang

Zhongshan Hospital Fudan University

Lei Yan

Zhongshan Hospital Fudan University

Zhongshu Kuang

Zhongshan Hospital Fudan University

Mingming Xue

Zhongshan Hospital Fudan University

Si Sun

Zhongshan Hospital Fudan University

Hao Xiang

Zhongshan Hospital Fudan University

Yanyan Hu

Zhongshan Hospital Fudan University

Zhimin Dong

Zhongshan Hospital Fudan University

Chaoyang Tong

Zhongshan Hospital Fudan University

Chunxue Bai

Zhongshan Hospital Fudan University

zhenju song ( $\nabla$ zhenjusong@yahoo.com )

Zhongshan Hospital Fudan University https://orcid.org/0000-0001-9294-8824

\section{Research}

Keywords: Sepsis, Immune dysfunction, Immune status classification, Cytokines, HLA-DR, Prognosis 
Posted Date: January 8th, 2020

DOI: https://doi.org/10.21203/rs.2.20262/v2

License: (1) This work is licensed under a Creative Commons Attribution 4.0 International License. Read Full License

Version of Record: A version of this preprint was published at World Journal of Emergency Medicine on January 1st, 2021. See the published version at https://doi.org/10.5847/wjem.j.1920-8642.2021.03.004. 


\section{Abstract}

\section{Background}

The dynamic monitoring of immune status is crucial to the precise and individualized treatment of sepsis. In this study, we introduced a model to describe and monitor the immune status of sepsis and explored its prognostic value.

Methods A prospective observational study was carried out in Zhongshan Hospital, Fudan University, enrolling septic patients admitted between July 2016 and December 2018. Blood samples were collected at day 1 and 3. Serum cytokines levels (TNF-a, IL-2R, IL-6, IL-8 and IL-10) and CD14 + monocyte HLA-DR expression were measured to serve as immune markers. Cut-off values were acquired from ROC curves and serum cytokine level higher than or HLA-DR level lower than cut point was defined as "positive", otherwise defined as "negative". Classification of each immune status, namely SIRS, CARS and MARS, was defined by numbers of positive immune markers levels. Changes of immune status from day 1 to 3 were classified into four groups which were Stabilization (SB), Deterioration (DT), Remission (RM) and Non-remission (NR).

Results

A total of 174 septic patients were enrolled including 50 non-survivors. In univariate analysis, serum levels of TNF-a, IL-2R, IL-6, IL-8 and IL-10 and HLA-DR expression at day 1 and 3 were associated with prognosis. Multivariate analysis discovered IL-10 and HLA-DR expression at day 3 were independent prognostic factors. Patients with MARS at day 1 had the highest mortality rate up to $58.7 \%$ among three immune status classifications, so did the patient with MARS at day 3 with mortality rate up to $69.2 \%$. Immune status of $46.1 \%$ patients changed from day 1 to 3 . Among four groups of immune status changes, DT had the highest mortality rate up to $77.8 \%$ followed by NR, RM and SB with mortality rate of $64.7 \%, 42.9 \%$ and $11.2 \%$ respectively.

\section{Conclusions}

Patients with severe immune disorder defined as MARS or deterioration of immune status defined as DT have the worst outcomes. The preliminary model of the classification and dynamic monitoring of immune status based on immune markers levels has prognostic values and worthy of further investigation.

Trail Registration: ChiCTR-OCC-14005202 (Chinese Clinical Trial Registry), Registered 09 September 2014, http://www.chictr.org.cn/showproj.aspx?proj=4373

\section{Background}

Sepsis is a common complication in patients with trauma, burns, infection, ischemia-reperfusion and surgery. It was defined as a dysregulated host response to infection and other injury factors [1,2]. The 
incidence and prevalence of sepsis have increased globally over the recent years and sepsis accounted for a considerable number of intensive care unit (ICU) admission and mortality [3]. Although the pathogenesis of sepsis is not fully explored, it's acknowledged that immune dysfunction and its damage to organ function added to the difficulty of disease management and thus influence the prognosis $[4,5]$.

Inflammatory activities of sepsis involved the interactions between various inflammatory mediators. The early phase of sepsis features activated inflammation process caused by systemic release of proinflammatory mediators [6]. Sepsis could also lead to the apoptosis and autophagy of immune cells, endotoxin tolerance and relevant center nervous system regulation, which consequently presented as immunosuppression [7]. Immunosuppression is more observed in severe patients as a result of imbalance in pro- and anti-inflammatory activities [6].

To describe the complex inflammatory process of sepsis, some definitions of immune status classification have been introduced in recent years. The process of sepsis features initial systemic inflammation process called systemic inflammatory response syndrome (SIRS). Anti-inflammatory activities may happen subsequently or concurrently and this compensatory response was called compensatory anti-inflammatory response syndrome (CARS) [8]. At times, an excessive response with both pro- and anti-inflammatory reactions called mixed antagonistic response syndrome (MARS) was found in some patients with severe dysfunction of immunomodulation $[9,10,11]$. Immune modulatory therapy was a research highlight of the treatment of sepsis, the changing of immune status and lack of specific clinical signs withheld the proper application of the therapy nevertheless. It was suggested that the precise identification of immune status would be the first step to provide appropriate immune therapies $[12,13]$.

Serum cytokines levels have been widely measured by clinicians to monitor immune status. Proinflammatory cytokines such as tumor necrosis factor-a (TNF-a), interleukin-2 receptor (IL-2R), IL-6 and IL8 and anti-inflammatory cytokines such as IL-10 were mostly adopted [14]. CD14 ${ }^{+}$monocyte human leukocyte antigen-D related (HLA-DR) expression is another biomarker of immune status and proven to have a high sensitivity and specificity. Monocytes with low HLA-DR expression have reduced ability of cytokine secretion and antigen presentation. It's considered that HLA-DR expression $<30 \%$ is indicative of immunosuppression $[15,16]$. Although those biomarkers can reflect the immune dysfunction and might have link with prognosis, single biomarker could not precisely reflect immune status due to the complexity of immune reactions. Thus, an immune model based on the combination of multiple biomarkers are needed.

In this study, we aimed to introduce a model of immune status classification of sepsis based on immune markers and to explore the association between the immune classification and prognosis. Firstly, we selected the most commonly used biomarkers in clinical practice such as cytokines (TNF-a, IL-2R, IL-6 and IL-8 and IL-10) and HLA-DR to explore the prognostic value of each biomarker. Secondly, we combined those biomarkers to introduce the model based on the definition of SIRS, CARS and MARS and compared the mortality of each classification. Thirdly, we introduced another model to describe the 
dynamic changes of immune status and also explored its prognostic value. Meeting abstract of this study has been published in Page S121, Volume 74 (Issue 4) of Annals of Emergency Medicine [17].

\section{Methods}

\section{Study setting and population}

We performed a prospective observational study in septic patients admitted to the ICU of Department of Emergency Medicine, Zhongshan Hospital, Fudan University between July 2016 and December 2018. The diagnosis of sepsis was according to the Third International Consensus Definitions for Sepsis and Septic

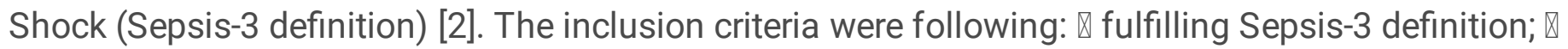
patients or authorized family members signed informed consent; $₫$ aged $\geq 18$ years old. The exclusion

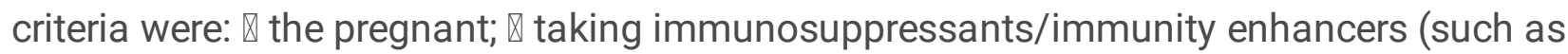
glucocorticoids, thymidine, recombinant human granulocyte stimulating factor) for more than 6 months prior to admission; $\otimes$ immunocompromised hosts such the recipients of chemotherapy or radiation therapy, the recipients of organ transplant and the patients infected by human immunodeficiency virus

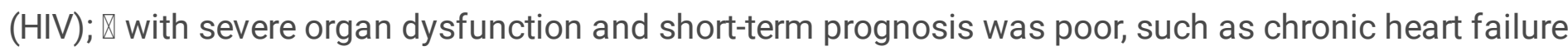
(New York Heart Assessment - IV), liver failure (Child-Pugh C) or kidney failure (Stage 5 Chronic Kidney Disease); $\otimes$ with history of mental illness. The clinical study protocol was approved by the Ethics Committee of Zhongshan Hospital, Fudan University (Approval number: B2014-082) and registered at Chinese Clinical Trial Registry (Registration number: ChiCTR-OCC-14005202).

\section{Data and sample collection}

The following clinical data were collected: $\otimes$ baseline characteristics such as age, gender and

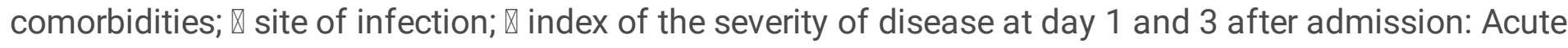
Physiology and Chronic Health Evaluation II (APACHE II) score and Sequential Organ Failure Assessment (SOFA) score; $\mathbb{Q}$ levels of inflammatory markers at day 1 and 3 after admission: peripheral white blood cell count (WBC), C-reaction protein (CRP) and procalcitonin (PCT); $\$ clinical interventions such as the use of glucocorticoids (equivalent to Prednisone $>200 \mathrm{mg} / \mathrm{d}$ ), mechanical ventilation, deep venous catheterization and urinary catheterization; $₫$ ICU length of stay (LOS) and outcome of hospital stay such as survival or death. Peripheral blood samples were collected at day 1 and 3 after admission in order to measure serum cytokines levels and HLA-DR expression. Due to the limit of practical clinical conditions, blood samples were collected at a part of patients at day 3 .

\section{Measurement of the levels of immune markers}

In order to measure serum cytokines levels, blood samples were collected in BD Vacutainer ${ }^{\circledR} \mathrm{SST}^{\mathrm{TM}}$ ॥ Advance tubes (BD Biosciences, CA, USA) at day 1 and 3 after admission. Plasma samples were preserved at $-80^{\circ} \mathrm{C}$ after centrifugation. The serum levels of TNF-a, IL-2R, IL-6, IL-8 and IL-10 were 
measured by ELISA (R\&D Systems, USA) according to manufacturer's instructions. To explore CD14 ${ }^{+}$ HLA-DR ${ }^{+}$monocytes expression, blood samples were collected in BD Vacutainer ${ }^{\circledR}$ K2 EDTA tubes at day 1 and 3 after admission. A following double color staining was utilized according to manufacturer's instructions: a fluorescein conjugated (FITC)-CD14 and an allophycocyanin conjugated (APC)-HLA-DR (BD Biosciences, CA, USA). Appropriate isotype controls were run with healthy controls and used for compensation and gating blood samples. Subsequently, samples were analyzed on an 18-parameter BD LSRFortessa machine with FlowJo software (Tree Star Inc, OR, USA). HLA-DR expression was expressed as the percentage of $\mathrm{CD} 14^{+} \mathrm{HLA}-\mathrm{DR}^{+}$monocyte among all $\mathrm{CD} 14^{+}$monocyte.

\section{Models of immune status classification and its dynamic changes}

We utilized the results of the measurement of each immune marker to define the immune status classification. TNF-a, IL-2R, IL- 6 and IL-8 were treated as pro-inflammatory markers while IL-10 and HLADR were anti-inflammatory markers. Cut-off values were acquired from Receiver Operating Characteristic (ROC) curves. Serum cytokine level higher than cut-off point or HLA-DR expression lower than cut-off point was defined as "positive (+)", otherwise defined as "negative (-)". SIRS was defined as 0-4 proinflammatory markers $(+)$ and 0 anti-inflammatory markers (+). CARS was defined as $0-2$ proinflammatory markers $(+)$ and 1-2 anti-inflammatory marker $(+)$. MARS was defined as 3-4 proinflammatory markers $(+)$ and 1-2 anti-inflammatory marker $(+)$ (Table 1 ). The dynamic changes of immune status from day 1 to 3 were classified into 4 following groups. Stabilization (SB) means that SIRS or CARS remained unchanged through day 1 to 3 . Deterioration (DT) means the change from SIRS or CARS at day 1 to MARS at day 3. Remission (RM) means the change from MARS at day 1 to SIRS or CARS at day 3. Non-remission (NR) means that MARS remained unchanged through day 1 to 3 (Table 2).

\section{Statistical analysis}

The Kolmogorov-Smirnov test was used to verify data normality. Normally distributed data were expressed as means \pm standard deviation (SD) and compared by Student's $t$ test. Abnormally distributed continuous variables were expressed as median (the 25th and 75th quartiles) and compared by MannWhitney $U$ test. Categorical data were expressed as frequency and percentage and compared by Pearson's chi-square test or Fisher's exact test when appropriate.

The risk factors of in-hospital mortality were explored by univariate analysis at first. Immune markers levels were among covariates, together with age, gender, comorbidities, main site of infection, severity of disease, inflammatory markers levels, clinical interventions and ICU LOS. Covariates with statistical significance were tested in multivariate logistic regression analysis by "Enter" method to explore independent risk factors. 
ROC curve was used to detect the cut-off value of each immune marker. Kaplan-Meier survival analysis was conducted to compare the outcomes between patients with positive and negative markers levels and between each classification of immune status and immune status changes based on a 30-day follow-up. Dynamic changes of inflammatory markers and disease severity scores of diseases from day 1 to 3 were tested by Wilcoxon signed rank test. The mortality rate of each immune status was compared by Pearson's chi-square test or Fisher's exact test when appropriate, so was that of each classification of immune status changes. All statistical analyses were two-sided, and the significance level was set to $P<$ 0.05. Model assumptions were tested before using statistical methods. Statistical analysis was conducted on SPSS 25.0 (SPSS Inc., IL, USA).

\section{Results}

\section{Characteristics of septic patients}

A total of 174 septic patients were enrolled in this study, including 124 survivors and 50 non-survivors. Data of all patients were available at day 1 . Six patients died before day 3 and one patient was discharged alive before day 3 . Blood drawing of 13 patients were not ordered by treating clinicians due to practical clinical conditions. Thus, data of 154 patients were available at day 3 including 115 survivors and 39 non-survivors.

A flowchart to illustrate recruitment process was shown in Fig 1. Among all enrolled patients, 109 were men and median age was 67-year-old. Respiratory tract was the most common site of primary infection $(n=117,62 \%)$. Other sites of infection included abdomen $(n=38,21.8 \%)$, urinary tract $(n=8,5 \%)$, skin and soft tissue $(n=6,3.4 \%)$ and blood stream $(n=5,2.9 \%)$. In total, $28 \%(50 / 174)$ of patients died in hospital. At day 1 , APACHE II score $(P<0.001)$, SOFA score $(P<0.001)$ and WBC $(P=0.024)$ were higher in non-survivors. At day 3 , APACHE II score $(P<0.001)$, SOFA score $(P<0.001)$, WBC $(P=0.024), \mathrm{CRP}(P=$ $0.002)$ and $\operatorname{PCT}(P<0.001)$ were all higher in non-survivors. The use of glucocorticoids $(P=0.007)$, mechanical ventilation $(P<0.001)$, deep venous catheterization $(P<0.001)$ and urinary catheterization $(P$ $<0.001)$ were more common in non-survivors. Also, survivors had longer in-hospital LOS $(P=0.002)$. The baseline characteristics of enrolled patients were shown in Table 3.

\section{Association between immune markers and in-hospital mortality of septic patients}

The results of the measurement of immune markers were shown in Table 4. The data revealed that proand anti- inflammatory activities were both the most serious at initial phase of sepsis, as serum levels of all cytokines were the highest at day 1 in both survivors and non-survivors. Univariate analysis revealed that non-survivors had higher levels of both pro- and anti- inflammatory cytokines and lower HLA-DR expression at day 1 (TNF-a: $P=0.003$, IL-2R: $P=0.013$, IL-6: $P<0.001$, IL-8: $P<0.001$, IL-10: $P<0.001$, HLA-DR: $P<0.001$ ) and 3 (TNF-a: $P<0.001$, IL-2R: $P=0.029$, IL-6: $P<0.001$, IL-8: $P<0.001$, IL-10: $P<$ 
0.001, HLA-DR: $P<0.001$ ). Cut-off values for each immune marker were calculated (Day 1: TNF-a: 19.95 pg/ml, IL-2R: 1684 pg/ml, IL-6: 39 pg/ml, IL-8: 20.75pg/ml, IL-10: 7.45 pg/ml, HLA-DR: 28.87\%; Day 3: TNF-a: 12.95 pg/ml, IL-2R: 1074.5 pg/ml, IL-6: 14.75 pg/ml; IL-8: 28.15 pg/ml; IL-10: 10.95 pg/ml; HLADR: $25.55 \%$ ) (Table 4). Patients with positive markers (except IL-2R at day 1 ) showed an worse 30-day survival as revealed by Kaplan-Meier survival analyses and Log-rank tests after applying cut-off values (Day 1: TNF-a: $P=0.035$, IL-6: $P=0.002$, IL-8: $P=0.02$, IL-10: $P=0.001$, HLA-DR: $P<0.001$; Day 3: TNF-a, $P<0.001$, IL-2R: $P<0.001$, IL-6: $P=0.013$; IL-8: $P=0.003$; IL-10: $P<0.001$; HLA-DR: $P<0.001$ ) (Fig 2 and Additional file 1: Fig S1). Multivariate analysis discovered that the levels of HLA-DR and IL-10 at day 3 were among independent risk factors of in-hospital mortality $(P=0.001$ and $P=0.018)$ (Table 5). The areas under ROC curve (AUCs) in the analysis of HLA-DR expression (AUC $=0.819, P<0.001)$ and IL-10 $(A \cup C=0.808, P<0.001)$ at day 3 were also higher than other markers (Table 4).

\section{Association between immune status classification and mortality of septic patients}

Since TNF-a, IL-2R, IL-6, IL-8, IL-10 and HLA-DR all had prognostic values in univariate analysis, we combined these markers to classify patients into certain immune status groups and explored their prognosis. The immune status classifications were based on the best cut-off values of serum levels of those immune markers as was introduced in Table 1.

Based on the immune status at day 1 and 3, patients classified into MARS had balanced baseline characteristics with those classified into SIRS and CARS, but higher disease severity scores (Day 1: APACHE II score: $P<0.001$, SOFA score: $P<0.001$; Day 3: APACHE II score: $P<0.001$, SOFA score: $P<$ 0.001 ) and higher levels of inflammatory markers (Day 1: WBC: $P<0.001$, CRP: $P<0.001$, PCT: $P<0.001$; Day 3: WBC: $P=0.005$, CRP: $P=0.001$, PCT: $P<0.001$ ) (see Additional file 2: Table $\mathbf{S 1}$ and Additional file

3: Table S2). MARS was associated with higher mortality rate of septic patients than SIRS and CARS (Day 1: OR 6.487, 95\% Cl 3.094 to 13.601, $P<0.001$; Day 3: OR 11.464, 95\% Cl 4.411 to 29.798, $P<0.001$ ) (Table 6). In survival analysis based on immune status at day 3, CARS and MARS both had worse prognosis than SIRS $(P<0.001$ and $P<0.001)$. It was also discovered that MARS had worse prognosis than CARS $(P=0.008)$ (Fig 2).

Immune status of 71 patients (46.1\%) changed from day 1 to 3 . To better describe the dynamic changes, four classifications were introduced. One hundred and seven patients were classified into SB group with a mortality of $11.2 \%$. Nine patients were classified into DT group with a mortality rate of $77.8 \%$, which was the highest. Twenty-one patients were classified into RM group and 17 patients were classified into NR group, with mortality rates of $42.9 \%$ and $64.7 \%$ respectively. APACHE II score $(P<0.001)$, SOFA score $(P<$ $0.001)$, WBC $(P=0.02)$, CRP $(P<0.001)$ and PCT $(P<0.001)$ decreased from day 1 to 3 in SB group (Table 7). However, no statistical significance was found in three other groups with regard to the corresponding changes in disease severity scores and inflammatory markers. In survival analysis, SB had better prognosis than RM, NR and DT $(P=0.028 ; P<0.001 ; P<0.001)$. 


\section{Discussion}

Immune mechanism of sepsis has been widely studied. Firstly, innate immune function was compromised due to the dysfunction of neutrophils, monocytes, dendritic cells and myeloid-derived suppressor cells (MDSCs) which subsequently altered first-line defense, inhibited T cell proliferation, altered inflammatory response and caused incomplete activation of T cells. Secondly, sepsis also influenced the effector functions and phenotypes of T cells, B cells and innate-type lymphocytes and thus compromised adaptive immune function [6]. Classic biphasic model presumed that sepsis featured initial pro-inflammatory phase and subsequent anti-inflammatory phase [18]. However, more evidence showed that sepsis-induced immune dysfunction are highly variable and thus introduced competition model that pro- and anti-inflammatory activities were under dynamic equilibrium and mutual interaction [19-23]. Thus, the proper monitoring of immune status and reversal of immune disorder are crucial to the treatment of septic patients. Combining multiple immune markers to compensate the low prediction efficiency of single markers in previous studies, this study proved the poor prognosis of patients with severe immune dysfunction named MARS and the deterioration of immune status named DT. Also, the variability of immune status was justified as we found the immune status of $46.1 \%$ were changed from day 1 to 3 .

Immune status can be measured by many approaches [24]. In this study, we chose cytokine as a biomarker of immune dysfunction. Serum cytokines levels were higher in non-survivors as we found, which was consistent with previous knowledge that sepsis induced intensive host immune reaction to pathogens called "cytokine storm" and could lead to poor prognosis [14, 25, 26]. Significantly, we also found IL-10 level at day 3 was an independent prognostic factor. HLA-DR expression was another chosen biomarker of immune dysfunction, which could reflect the functions of both innate and adaptive immune system and its lower expression indicated immunosuppression $[6,15]$. In our study, non-survivors had lower HLA-DR expression at all points in time. The cut-off values were $28.87 \%$ and $25.55 \%$ at day 1 and 3 respectively. Both were close to the previously recognized value $30 \%$, which proved the reliability of our model. HLA-DR expression at day 3 was also an independent risk factor of poor outcome as revealed by multivariate analysis and had the highest prognostic value based on the data of AUC. This confirmed the previous concept that HLA-DR expression could best reflect the immune function and predict the prognosis after 48 hours of sepsis duration [16, 27]. Our study also justified the competition model by demonstrating that pro- and anti-inflammatory activities in sepsis occurred concurrently which were reflected by simultaneously elevated serum levels of both pro- and anti-inflammatory cytokines and lower HLA-DR expression. The independent mortality predictability of two immunosuppressive markers (HLADR and IL-10 at day 3) revealed by multivariate analysis added to the importance of monitoring immunosuppressive activities.

Although the prognostic values of many immune markers have been widely studied, it was rarely reported how to combine multiple immune markers to reach a more precise definition of immune status and prediction of mortality. A previous study designed and analyzed prognostic value of the combined scores of anti-and pro- inflammatory cytokines in septic patients and proved its higher value than each single 
biomarker [28]. In this study, we combined HLA-DR expression and cytokines (TNF-a, IL-2R, IL-6, IL-8 and IL-10) to quantitatively define each immune status, namely SIRS, CARS and MARS. A recent study on mechanisms in mice and human serum confirmed that CARS could arise at the beginning of disease course but not following SIRS. SIRS and CARS were both regulated by infiltrating macrophages and T cells and dependent on NLRP 3 inflammasome. SIRS was initiated by the activation of macrophages and CARS was regulated by T cell function [29]. The concept of MARS was introduced as either the coexistence of overwhelming inflammation and suppression of innate and adaptive immunity or an intermediate phase between SIRS and CARS $[11,30]$. Based on the numbers of "positive" immune markers, we defined SIRS as moderate inflammatory process without corresponding anti-inflammatory process. CARS was defined as simultaneous moderate pro- and anti- inflammatory processes. We defined MARS as an independent phase of severe imbalanced pro- and anti- inflammatory processes, rather than a transit between SIRS and CARS.

Our data demonstrated MARS was more frequent in non-survivors while SIRS and CARS were more frequent in survivors. This confirmed the assumption that the uncontrolled, excessive inflammatory activities could lead to poor prognosis.

High proportion (46.1\%) of the changes of immune status from day 1 to 3 was revealed by our study. It has been addressed by many previous studies that the changes of immune status could not been underestimated $[4,12,13]$, so we introduced an model which could dynamically monitor that change. As we found, DT had the highest mortality rate of $77.8 \%$ while SB had the lowest mortality rate of $11.2 \%$. It laid stress on the importance of maintaining the stable immune status for patients with SIRS or CARS on admission. Because the prognosis would take a turn for the worse once the immune status shifts into MARS. Mortality rate of RM and NR groups were both high but the former was comparatively lower, which suggested the proper management of immune disorder could bring survival benefit to the patients with MARS. In SB group, we discovered the corresponding decrease in disease severity scores and inflammatory markers levels. However, the corresponding changes were not statistical significant in other groups. This might be explained by the relatively small sample sizes of these groups or that immune status classification has higher sensitivity of predicting mortality than disease severity scores and inflammatory markers.

Our study had several limitations. First, the sample size was relatively small as a single-center study. Second, we only collected the data at day 1 and 3 . Thus the immune status and its dynamic changes could not be traced later than day 3 . Third, we acquired cut-off value of each immune marker from ROC curve to set a model of immune dysfunction, the rationale of this classification are worthy of further verification. Fourth, only 6 accessible biomarkers were chosen as elements of classification, more biomarkers with high sensitivity and specificity are needed to be discovered. Also, the use of glucocorticoids might influence the process of immune reaction and expression of immune markers but this condition was more similar to real clinical setting.

\section{Conclusions}


In conclusion, we confirmed that immune markers such as serum cytokines levels and HLA-DR expression were associated with prognosis of septic patients. Patients with severe immune disorder defined as MARS or deterioration of immune status defined as DT were more likely to have poor outcome. As the concept of stage-adjusted therapy for sepsis is promising, our study has provided a preliminary model for the monitoring of immune status which might be beneficial to the individualized treatments for patients.

\section{Abbreviations}

APACHE II Acute Physiology and Chronic Health Evaluation II

APC Allophycocyanin

AUC Area under curve

CARS Compensatory anti-inflammatory response syndrome

CRP C-reaction protein

DT Deterioration

FITC Fluorescein-5-isothiocyanate

HLA-DR Human leukocyte antigen-D related

ICU Intensive care unit

IL Interleukin

LOS Length of stay

MARS Mixed antagonistic response syndrome

MDSC Myeloid-derived suppressor cell

NR Non-remission

PCT Procalcitonin

RM Remission

ROC Receiver operating characteristic

SB Stabilization

SD Standard deviation 
SIRS Systemic inflammatory response syndrome

SOFA Sequential Organ Failure Assessment

TNF Tumor necrosis factor

WBC White blood cell count

\section{Declarations}

\section{Ethics approval and consent to participate}

The clinical study protocol was approved by the Ethics Committee of Zhongshan Hospital, Fudan University (Approval number: B2014-082). Informed consent was obtained from each participant or legal surrogate.

\section{Consent for publication}

All authors give their consent for publication.

\section{Availability of data and materials}

The datasets used and/or analyzed during the current study are available from the corresponding authors on reasonable request.

\section{Competing interests}

The authors have no competing interests to declare.

\section{Funding}

This work was supported by National Natural Science Foundation of China $(81471840,81171837)$, Shanghai Traditional Medicine Development Project (ZY3-CCCX3-3018, ZHYY-ZXYJH-201615), Key Project of Shanghai Municipal Health Bureau (2016ZB0202).

\section{Authors' contributions}

ZS, CB, JY, JH , YC and CT contributed to the conception and design of the work. JY, YC, ZK, MX, SS, HX, $\mathrm{YH}$ and ZD contributed to the data collection, statistical analysis and the interpretation of the results. JH, LY and ZK performed the experimental analyses of measurements of HLA-DR expression and serum cytokines levels. YC, JY, CB and ZS contributed to the drafting and revision of the manuscript. All authors have approved the final version of the manuscript.

\section{Acknowledgements}

Not applicable. 


\section{Authors' information}

${ }^{1}$ Department of Emergency Medicine, Zhongshan Hospital, Fudan University, Shanghai, 200032, China

2 Department of Respiratory and Critical Care Medicine, Zhongshan Hospital, Fudan University, Shanghai, 200032, China

\section{References}

[1] Kempker JA, Martin GS. The Changing Epidemiology and Definitions of Sepsis. Clin Chest Med. 2016;37(2):165-79.

[2] Singer M, Deutschman CS, Seymour CW, et al. The Third International Consensus Definitions for Sepsis and Septic Shock (Sepsis-3). JAMA. 2016;315(8):801-10.

[3] Perner A, Gordon AC, De Backer D, et al. Sepsis: frontiers in diagnosis, resuscitation and antibiotic therapy. Intensive Care Med. 2016; 42(12):1958-69.

[4] Hotchkiss RS, Monneret G, Payen D. Sepsis-induced immunosuppression: from cellular dysfunctions to immunotherapy. Nat Rev Immunol. 2013;13(12):862-74.

[5] Chen XH, Yin YJ, Zhang JX. Sepsis and immune response. World J Emerg Med. 2011;2(2):88-92.

[6] Venet F, Monneret G. Advances in the understanding and treatment of sepsis-induced immunosuppression. Nature Reviews Nephrology 2017;14(2):121-37.

[7] Gurung P, Rai D, Condotta SA, Babcock JC, Badovinac VP, Griffith TS. Immune unresponsiveness to secondary heterologous bacterial infection after sepsis induction is TRAlL dependent. J Immunol. 2011;187(5):2148-54.

[8] Ward NS, Casserly B, Ayala A. The compensatory anti-inflammatory response syndrome (CARS) in critically ill patients. Clin Chest Med. 2008;29(4):617-25.

[9] Kox WJ, Volk T, Kox SN, Volk HD. Immunomodulatory therapies in sepsis. Intensive Care Med. 2000;26 Suppl 1:S124-8. [10] Hotchkiss RS, Monneret G, Payen D. Immunosuppression in sepsis: a novel understanding of the disorder and a new therapeutic approach. Lancet Infect Dis. 2013;13(3):260-8.

[11] Novotny AR, Reim D, Assfalg V, et al. Mixed antagonist response and sepsis severity-dependent dysbalance of pro- and anti-inflammatory responses at the onset of postoperative sepsis. Immunobiology. 2012;217(6):616-21.[12] Venet F, Lepape A, Monneret G. Clinical review: flow cytometry perspectives in the ICU - from diagnosis of infection to monitoring of injury-induced immune dysfunctions. Crit Care. 2011;15(5):231. 
[13] Venet F, Lukaszewicz AC, Payen D, Hotchkiss R, Monneret G. Monitoring the immune response in sepsis: a rational approach to administration of immunoadjuvant therapies. Curr Opin Immunol. 2013;25(4):477-83.

[14] Mera S, Tatulescu D, Cismaru C, et al. Multiplex cytokine profiling in patients with sepsis. APMIS. 2011;119(2):155-63.

[15] Volk HD, Reinke P, Krausch D, et al. Monocyte deactivation-rationale for a new therapeutic strategy in sepsis. Intensive Care Med. 1996;22 Suppl 4:S474-81.

[16] Monneret G, Lepape A, Voirin N, et al. Persisting low monocyte human leukocyte antigen-DR expression predicts mortality in septic shock. Intensive Care Med. 2006;32(8):1175-83.

[17] Chen Y, Song Z. The association between immune dysfunction and outcome of septic patients: a retrospective observational study. Ann Emerg Med. 2019;74(4):S121.

[18] Muenzer JT, Davis CG, Chang K, et al. Characterization and modulation of the immunosuppressive phase of sepsis. Infect Immun. 2010;78(4):1582-92.

[19] Tang BM, Huang SJ, McLean AS. Genome-wide transcription profiling of human sepsis: a systematic review. Crit Care. 2010;14(6):R237.

[20] Yende S, Kellum JA, Talisa VB, et al. Long-term host immune response trajectories among hospitalized patients with sepsis. JAMA Netw Open. 2019;2(8):e198686.

[21] Munford RS, Pugin J. Normal responses to injury prevent systemic inflammation and can be immunosuppressive. Am J Respir Crit Care Med. 2001;163(2):316-21.

[22] Stearns-Kurosawa DJ, Osuchowski MF, Valentine C, Kurosawa S, Remick DG. The pathogenesis of sepsis. Annu Rev Pathol. 2011; 6:19-48.

[23] Tamayo E, Fernández A, Almansa R, et al. Pro- and anti- inflammatory responses are regulated simultaneously from the first moments of septic shock. Eur Cytokine Netw. 2011;22(2):82-7.

[24] Reinhart K, Bauer M, Riedemann NC, Hartog CS. New approaches to sepsis:molecular diagnostics and biomarkers. Clin Microbiol Rev. 2012;25(4):609-34.

[25] Panacek EA, Marshall JC, Albertson TE, et al. Monoclonal Anti-TNF: a Randomized Controlled Sepsis Study Investigators. Efficacy and safety of the monoclonal anti-tumor necrosis factor antibody $F\left(a b^{\prime}\right) 2$ fragment afelimomab in patients with severe sepsis and elevated interleukin-6 levels. Crit Care Med. 2004;32(11):2173-82.

[26] Heper Y, Akalin EH, Mistik R, et al. Evaluation of serum C-reactive protein, procalcitonin, tumor necrosis factor alpha, and interleukin-10 levels as diagnostic and prognostic parameters in patients with 
community-acquired sepsis, severe sepsis, and septic shock. Eur J Clin Microbiol Infect Dis. 2006;25(8):481-91.

[27] Drewry AM, Ablordeppey EA, Murray ET, et al. Comparison of monocyte human leukocyte antigen-DR expression and stimulated tumor necrosis factor alpha production as outcome predictors in severe sepsis: a prospective observational study. Crit Care. 2016;20(1):334.

[28] Andaluz-Ojeda D, Bobillo F, Iglesias V, et al. A combined score of pro- and anti-inflammatory interleukins improves mortality prediction in severe sepsis. Cytokine. 2012;57(3):332-6.

[29] Sendler M, van den Brandt C, Glaubitz J, et al. NLRP3 Inflammasome Regulates Development of Systemic Inflammatory Response and Compensatory Anti-inflammatory Response Syndromes in Mice With Acute Pancreatitis. Gastroenterology. 2020;158(1):253-269.e14.

[30] Osuchowski MF, Welch K, Siddiqui J, Remick DG. Circulating cytokine/inhibitor profiles reshape the understanding of the SIRS/CARS continuum in sepsis and predict mortality. J Immunol. 2006;177(3):1967-74.

\section{Additional Files}

Additional file 1: Fig S1. Kaplan-Meier survival curves of septic patients. Comparisons between the group with positive immune marker level and that with negative level are shown in panel (A)-(J). (A) TNF- $a$ at day 1. $P=0.035$; (B) TNF-a at day 3. $P<0.001$; (C) IL-2R at day $1 . P=0.068$; (D) IL-2R at day 3. $P<0.001$; (E) IL-6 day 1. $P=0.002 ;(\mathrm{F}) \mathrm{IL}-6$ at day 3. $P=0.013 ;(\mathrm{G}) \mathrm{IL}-8$ at day 1. $P=0.02 ;(\mathrm{H}) \mathrm{IL}-8$ at day $3 . P=$ 0.003; (I) IL-10 at day 1. $P=0.001$; (J) HLA-DR at day 1. $P<0.001$; Comparison between different immune status classifications at day 1 is shown in panel (K). (K) $P=0.0007$ (SIRS vs. CARS), $P<0.0001$ (SIRS vs. MARS), $P=0.0105$ (CARS vs. MARS).

Additional file 2: Table S1. Clinical and demographic characteristics of enrolled patients classified according to immune status at day 1 .

Additional file 3: Table S2. Clinical and demographic characteristics of enrolled patients classified according to immune status at day 3 .

\section{Tables}


Table 1 Immune status classification of septic patients

\begin{tabular}{ccc}
\hline Pro-inflammatory markers ${ }^{1}$ & Anti-inflammatory markers ${ }^{2}$ & Classification \\
\hline $0-2(+)$ & $(-)$ & SIRS \\
$3-4(+)$ & $(-)$ & \\
$0-2(+)$ & $1-2(+)$ & CARS \\
$3-4(+)$ & $1-2(+)$ & MARS \\
\hline
\end{tabular}

1 TNF-a, IL-2R, IL-6, IL-8

2 IL-10, HLA-DR

Table 2 Classifications of the immune status changes from day 1 to 3

\begin{tabular}{ccc}
\hline Day 1 & Day 3 & Classification \\
\hline SIRS or CARS & SIRS or CARS & Stabilization (SB) \\
& MARS & Deterioration (DT) \\
MARS & SIRS or CARS & Remission (RM) \\
& MARS & Non-remission (NR) \\
\hline
\end{tabular}


Table 3 Clinical and demographic characteristics of enrolled patients classified according to prognosis

\begin{tabular}{|c|c|c|c|}
\hline & $\begin{array}{c}\text { Survivors } \\
(\mathrm{n}=124)\end{array}$ & $\begin{array}{l}\text { Non-survivors } \\
(\mathrm{n}=50)\end{array}$ & $P$ value \\
\hline Age (years) & $67.5(48.25-78)$ & $67(56.75-73.25)$ & 0.703 \\
\hline Male (n, \%) & $78(62.9)$ & $31(62)$ & 0.911 \\
\hline \multicolumn{4}{|l|}{ Comorbidities, n (\%) } \\
\hline Hypertension & $58(46.8)$ & $25(50)$ & 0.7 \\
\hline Diabetes mellitus & $33(26.6)$ & $15(30)$ & 0.651 \\
\hline Coronary heart disease & $12(9.7)$ & $7(14)$ & 0.408 \\
\hline $\begin{array}{l}\text { Chronic obstructive pulmonary } \\
\text { disease }\end{array}$ & $10(8.1)$ & $5(10)$ & 0.91 \\
\hline Hepatitis and cirrhosis & $12(9.7)$ & $3(6)$ & 0.595 \\
\hline Renal insufficiency & $14(11.3)$ & $7(14)$ & 0.62 \\
\hline Malignancy & $11(8.9)$ & $6(12)$ & 0.729 \\
\hline \multicolumn{4}{|l|}{ Main site of infection, $\mathrm{n}(\%)$} \\
\hline Respiratory tract & $80(64.5)$ & $37(74)$ & 0.707 \\
\hline Abdomen & $30(24.2)$ & $8(16)$ & 0.311 \\
\hline Urinary tract & $6(4.8)$ & $2(4)$ & 1 \\
\hline Skin and soft tissue & $4(3.2)$ & $2(4)$ & 1 \\
\hline Blood stream & $4(3.2)$ & $1(2)$ & 1 \\
\hline \multicolumn{4}{|c|}{ Index of severity of disease, median (25th, 75 th) } \\
\hline \multicolumn{4}{|c|}{ APACHE II score } \\
\hline Day 1 & $12(7,16)$ & $20.5(11.75,26)$ & $<0.001$ \\
\hline Day $3^{1}$ & $9(6,13)$ & $18(14,25)$ & $<0.001$ \\
\hline \multicolumn{4}{|l|}{ SOFA score } \\
\hline Day 1 & $3(2,4)$ & $5(3.75,10.25)$ & $<0.001$ \\
\hline Day $3^{1}$ & $2(1,4)$ & $6(4,8)$ & $<0.001$ \\
\hline \multicolumn{4}{|c|}{ Inflammatory markers, median (25th, 75th) } \\
\hline \multicolumn{4}{|l|}{$\mathrm{WBC}, * 10^{\wedge} 9 / \mathrm{L}$} \\
\hline Day 1 & $\begin{array}{c}10.85(6.99 \\
14.5)\end{array}$ & $12.6(8.16,18.4)$ & 0.024 \\
\hline Day $3^{1}$ & $9.07(6.9,12.09)$ & $13.15(9.55,19.79)$ & $<0.001$ \\
\hline \multicolumn{4}{|l|}{$\mathrm{CRP}, \mathrm{mg} / \mathrm{L}$} \\
\hline Day 1 & $90(56.88,96)$ & $90(62.55,138.6)$ & 0.353 \\
\hline Day $3^{1}$ & $\begin{array}{l}52.2(15.2 \\
104.6)\end{array}$ & $90(66.1,143.5)$ & 0.002 \\
\hline \multicolumn{4}{|l|}{ PCT, ng/L } \\
\hline Day 1 & $1.14(0.29,5.07)$ & $1.47(0.4,10.4)$ & 0.172 \\
\hline Day $3^{1}$ & $0.56(0.15,1.98)$ & $1.85(0.6,11.13)$ & $<0.001$ \\
\hline \multicolumn{4}{|l|}{ Interventions, n (\%) } \\
\hline $\begin{array}{l}\text { Glucocorticoids (Prednisone > } \\
\text { 200mg) }\end{array}$ & $67(54)$ & $38(76)$ & 0.007 \\
\hline Mechanical ventilation & $62(50)$ & $46(92)$ & $<0.001$ \\
\hline Deep venous catheterization & $54(43.5)$ & $38(76)$ & $<0.001$ \\
\hline Urinary catheterization & $57(46)$ & $43(86)$ & $<0.001$ \\
\hline ICU length of stay (days) & $14(10,21.75)$ & $10(5,20.5)$ & $<0.002$ \\
\hline
\end{tabular}

${ }^{1}$ Data were available in 154 patients, including 115 survivors and 39 non-survivors. 
Table 4 Immune markers of septic patients

\begin{tabular}{|c|c|c|c|c|c|}
\hline & $\begin{array}{c}\text { Survivors } \\
(\mathrm{n}=124)\end{array}$ & $\begin{array}{c}\text { Non-survivors } \\
(\mathrm{n}=50)\end{array}$ & $P$ value & AUC & Cut-off value \\
\hline \multicolumn{6}{|c|}{ Serum cytokine level, $(\mathrm{pg} / \mathrm{ml}) \square$ Median (25th,75th) } \\
\hline \multicolumn{6}{|l|}{ TNF- $\alpha$} \\
\hline Day 1 & $12.1(9.1-16.8)$ & $17.1(11.2-25.3)$ & 0.003 & 0.648 & 17.95 \\
\hline Day $3^{1}$ & $11.3(8.6-12.9)$ & $12.7(11.1-21.4)$ & $<0.001$ & 0.735 & 12.95 \\
\hline \multicolumn{6}{|l|}{ IL-2R } \\
\hline Day 1 & 1092(735-1700) & $1543.5(892.3-2548.8)$ & 0.013 & 0.599 & 1684 \\
\hline Day 31 & $892(654-1036)$ & $1088(678-1898)$ & 0.029 & 0.615 & 1074.5 \\
\hline \multicolumn{6}{|l|}{ IL-6 } \\
\hline Day 1 & 24.4(9.6-81.8) & $68(18.4-212.3)$ & $<0.001$ & 0.654 & 39 \\
\hline Day $3^{1}$ & $13.1(6.2-28.1)$ & $23.1(13.1-101)$ & $<0.001$ & 0.717 & 14.75 \\
\hline \multicolumn{6}{|l|}{ IL-8 } \\
\hline Day 1 & $25(13.2-66.2)$ & $58.5(24.8-95.6)$ & $<0.001$ & 0.645 & 20.75 \\
\hline Day 31 & $21.1(11.2-35.3)$ & $32.5(23-83.2)$ & $<0.001$ & 0.695 & 28.15 \\
\hline \multicolumn{6}{|l|}{ IL-10 } \\
\hline Day 1 & $5.7(5-13)$ & $18.1(6.5-50.6)$ & $<0.001$ & 0.679 & 7.45 \\
\hline Day $3^{1}$ & $5(5-6.7)$ & $14(6.5-26.8)$ & $<0.001$ & 0.808 & 10.95 \\
\hline \multicolumn{6}{|c|}{ HLA-DR expression (\%)ロMedian(25th,75th) } \\
\hline Day 1 & $38.2(20.3-47.9)$ & $18.5(13.4-27.3)$ & $<0.001$ & 0.673 & 28.87 \\
\hline Day 31 & $44.4(29.3-56.4)$ & $16.9(12.2-28.1)$ & $<0.001$ & 0.819 & 25.55 \\
\hline
\end{tabular}

${ }^{1}$ Data were available in 154 patients, including 115 survivors and 39 non-survivors.

Table 5 Results of the multivariate logistic regression test of the risk factors of in-hospital mortality

\begin{tabular}{lcccccc}
\hline \multicolumn{1}{c}{ Variables ${ }^{1}$} & $\begin{array}{c}\text { Partial regression } \\
\text { coefficient }\end{array}$ & $\begin{array}{c}\text { Standard } \\
\text { error }\end{array}$ & $\begin{array}{c}\text { Wald } \\
\chi 2\end{array}$ & $P$ value & OR & $95 \%$ CI \\
\hline $\begin{array}{l}\text { Mechanical } \\
\text { ventilation }\end{array}$ & 3.47 & 1.408 & 6.078 & 0.014 & 32.14 & $\begin{array}{c}2.037- \\
\text { APACHE II score }\end{array}$ \\
$\begin{array}{l}\text { at day 3 } \\
\text { SOFA score at day }\end{array}$ & 0.297 & 0.109 & 7.4 & 0.007 & 1.346 & $1.086-$ \\
3 & -0.464 & 0.211 & 4.824 & 0.028 & 0.629 & $0.415-$ \\
CRP at day 3 & 0.01 & 0.005 & 4.372 & 0.037 & 1.01 & $1.001-$ \\
& & & & & & 1.020 \\
\hline HLA-DR at day 3 & -0.105 & 0.031 & 11.709 & 0.001 & 0.901 & $0.848-$ \\
& & & & & & 0.957 \\
\hline IL-10 at day 3 & 0.123 & 0.052 & 5.593 & 0.018 & 1.131 & $1.021-$ \\
& & & & & & 1.252 \\
\hline
\end{tabular}

${ }^{1}$ Analysis was conducted by means of Enter method. Other covariates without significance included all disease severity scores and inflammatory markers at day 1 , levels of WBC, PCT, TNF- $\alpha$, IL-2R, IL-6 and IL-8 at day 3, use of glucocorticoids, deep venous catheterization and urinary catheterization and ICU LOS. 
Table 6 Association between immune status classification and outcome of septic patients

\begin{tabular}{lcccccc}
\hline & Day 1 & \multicolumn{3}{c}{ Day 3 } \\
\hline & SIRS & CARS & MARS & SIRS & CARS & MARS \\
\hline Survivors, n(\%) ${ }^{1}$ & $57(98.3)$ & $48(68.6)$ & $19(41.3)$ & $83(94.3)$ & $24(60)$ & $8(30.7)$ \\
Non-survivors, n(\%) $^{1}$ & $1(1.7)$ & $22(31.4)$ & $27(58.7)$ & $5(5.7)$ & $16(40)$ & $18(69.2)$ \\
$P_{\mathrm{A}} / P_{\mathrm{B}}{ }^{2}$ & \multicolumn{2}{c}{$<0.001 / 0.004$} & & $<0.001 / 0.02$ & \\
\hline
\end{tabular}

${ }^{1}$ Percentage indicates the proportion of in-hospital survivors/non-survivors among each immune status.

${ }^{2} P_{\mathrm{A}}$ value stands for comparison between SIRS+CARS and MARS and $P_{\mathrm{B}}$ value for comparison between CARS and MARS.

Table 7 Prognosis of each group of immune status changes from day 1 to 3

\begin{tabular}{|c|c|c|c|c|}
\hline & $\begin{array}{c}\text { Stabilization } \\
\text { (SB) }\end{array}$ & $\begin{array}{c}\text { Deterioration } \\
\text { (DT) }\end{array}$ & $\begin{array}{c}\text { Remission } \\
\text { (RM) }\end{array}$ & $\begin{array}{c}\text { Non-remission } \\
\text { (NR) }\end{array}$ \\
\hline Survivors, n (\%) $^{1}$ & $95(88.8)$ & $2(22.2)$ & $12(57.1)$ & $6(35.3)$ \\
\hline $\begin{array}{l}\text { Non-survivors, } \mathrm{n} \\
(\%)^{1}\end{array}$ & $12(11.2)$ & 7 (77.8) & $9(42.9)$ & $11(64.7)$ \\
\hline \multicolumn{5}{|c|}{ Changes of index of severity of disease, median (25th, 75th) } \\
\hline $\begin{array}{l}\Delta \text { APACHE II } \\
\text { score }\end{array}$ & $-3(-6,0)^{2}$ & $0(-6,6.5)$ & $-1(-6.5,1)$ & $0(-5,1.5)$ \\
\hline$\Delta$ SOFA score & $-1(-1,0)^{2}$ & $1(1,2.5)$ & $-2(-3,-0.5)^{2}$ & $0(-0.5,1.5)$ \\
\hline \multicolumn{5}{|c|}{ Changes of inflammatory markers, median (25th, 75th) } \\
\hline$\Delta \mathrm{WBC}, * 10^{\wedge} 9 / \mathrm{L}$ & $\begin{array}{c}-0.58(-3.52 \\
1.49)^{2}\end{array}$ & $\begin{array}{c}0.15(-3.12 \\
15.07)\end{array}$ & $\begin{array}{c}-0.62(-8.22 \\
4.2)\end{array}$ & $\begin{array}{c}-0.64(-6.52, \\
2.02)\end{array}$ \\
\hline$\Delta \mathrm{CRP}, \mathrm{mg} / \mathrm{L}$ & $\begin{array}{c}-26.4(-67.5 \\
8.19)^{2}\end{array}$ & $\begin{array}{c}24.4(-44.5 \\
77.11)\end{array}$ & $\begin{array}{c}3(-52.15 \\
51.8)\end{array}$ & $0(-50.5,66)$ \\
\hline$\Delta \mathrm{PCT}, \mathrm{ng} / \mathrm{L}$ & $\begin{array}{c}-0.27(-2.28 \\
-0.01)^{2}\end{array}$ & $\begin{array}{c}0.17(-12.37 \\
1.42)\end{array}$ & $\begin{array}{c}-0.21(-1.64 \\
0.21)\end{array}$ & $\begin{array}{c}0.26(-6.65 \\
21.77)\end{array}$ \\
\hline$P_{\mathrm{A}} / P_{\mathrm{B}}{ }^{3}$ & \multicolumn{4}{|c|}{$<0.001 /<0.001$} \\
\hline
\end{tabular}

${ }_{1}^{1}$ Percentage indicates the proportion of in-hospital survivors/non-survivors among each immune status.

${ }^{2} P<0.05$.

${ }^{3} P_{\mathrm{A}}$ value stands for comparison between $\mathrm{SB}+\mathrm{RM}$ (with better prognosis) and DT+NR (with worse prognosis), $P_{\mathrm{B}}$ value for comparison between DT (with the worst prognosis) and other classification.

\section{Figures}




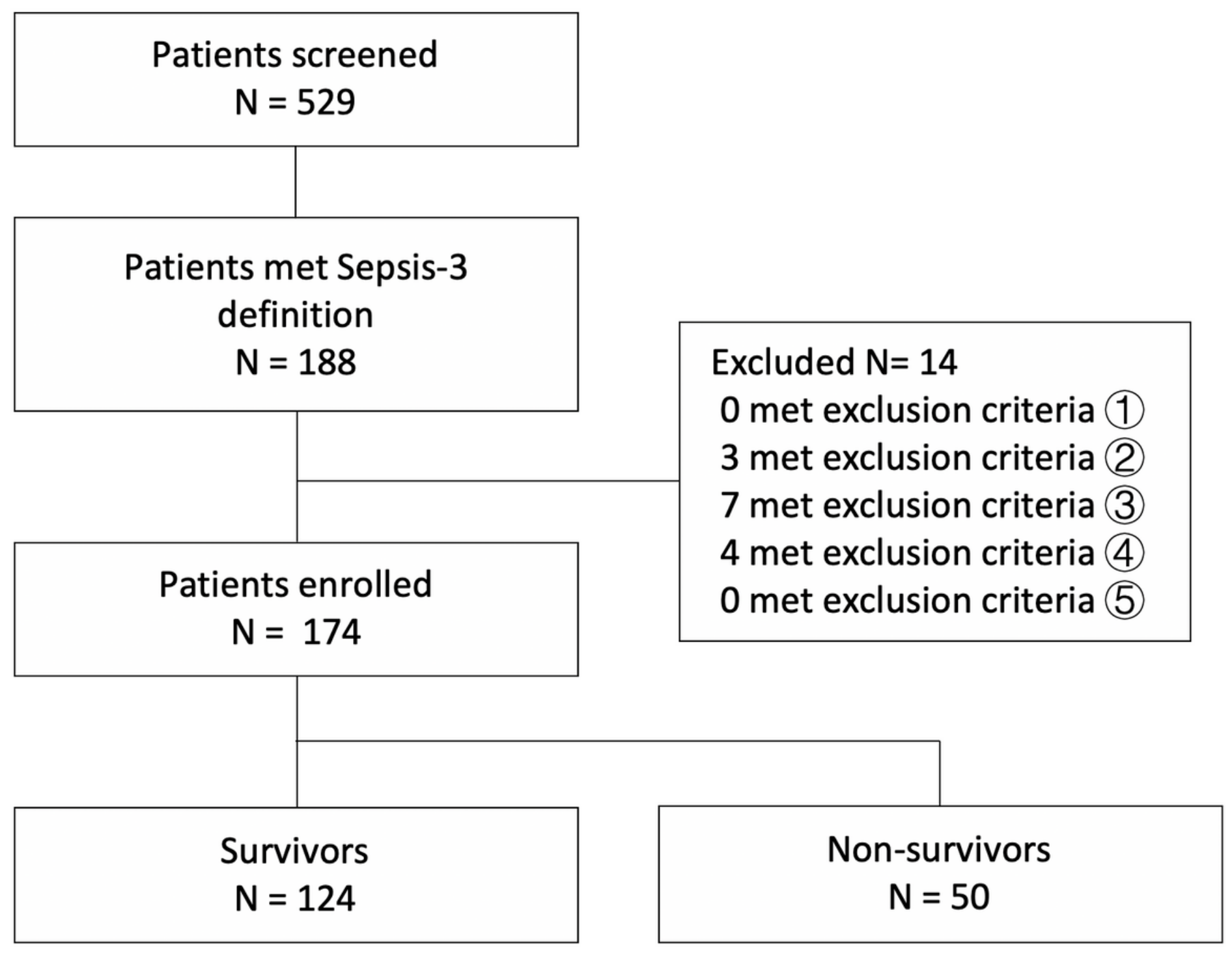

Figure 1

Study Flowchart. 
A

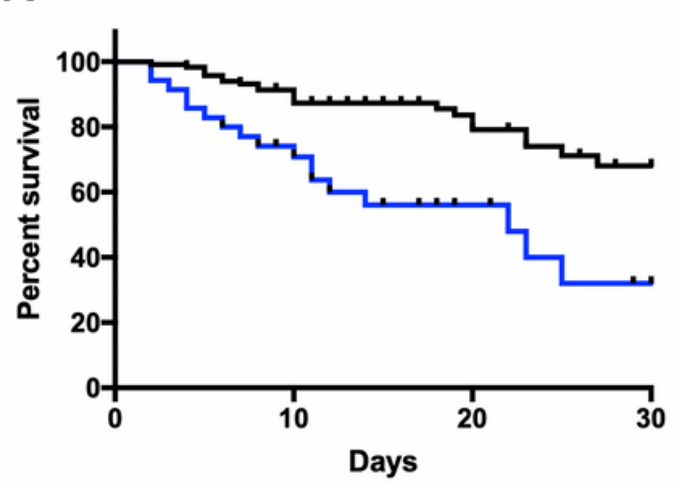

c

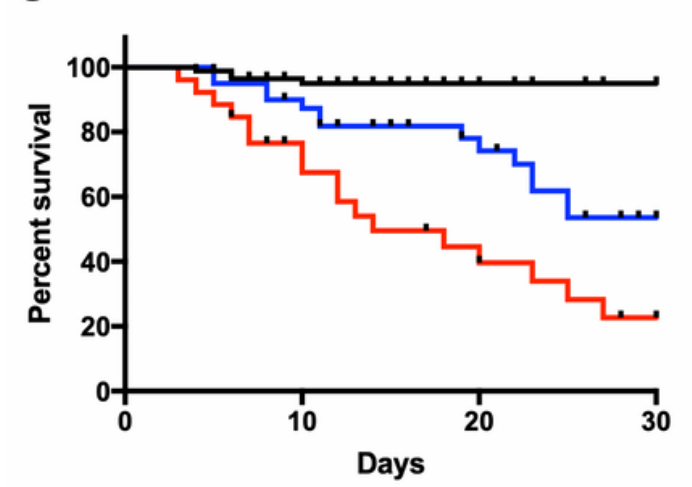

B

HLA-DR day 3
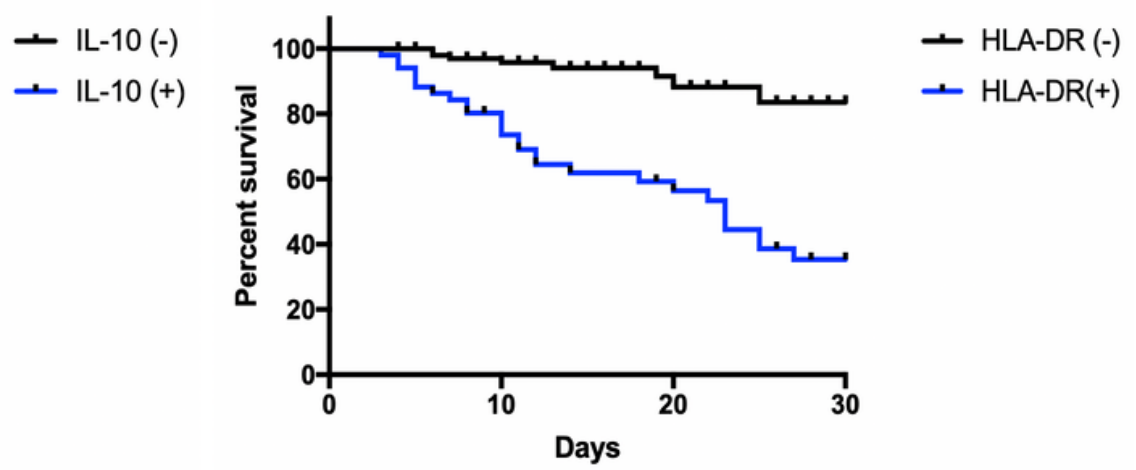

D Changes of immune status
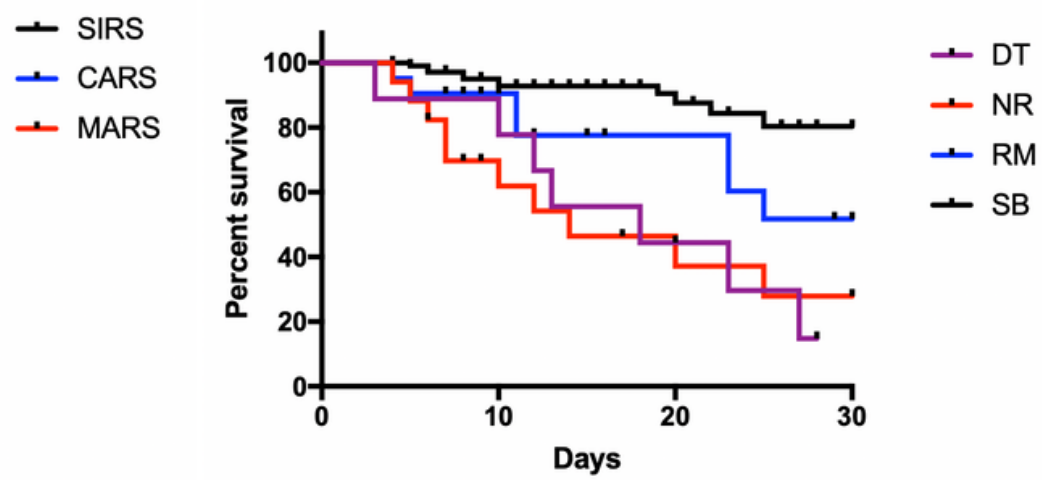

Figure 2

Kaplan-Meier survival curves of septic patients. (A) Comparison between the group with positive IL-10 level and that with negative level at day 3. $\mathrm{P}<0.001$; (B) Comparison between the group with positive HLA-DR level and that with negative level at day 3. $P<0.001$; (C) Comparison between different immune status classifications at day 3. $P=0.0007$ (SIRS vs. CARS), $P<0.001$ (SIRS vs. MARS). $P=0.008$ (CARS vs. MARS); (D) Comparison between different classifications of immune status changes. $P=0.028$ (SB vs. RM), $P<0.001$ (SB vs. NR), $P<0.001$ (SB vs. DT), $P=0.086$ (RM vs. RM). Survival curve of DT had intersections with that of RM and NR, in which case the Log-rank test was not conducted.

\section{Supplementary Files}

This is a list of supplementary files associated with this preprint. Click to download.

- Tables2.docx

- FigureS1.png

- TableS1.docx 